\title{
Entanglement and Coherence in Quantum State Merging
}

\author{
A. Streltsov, ${ }^{1,2, *}$ E. Chitambar, ${ }^{3}$ S. Rana, ${ }^{1}$ M. N. Bera, ${ }^{1}$ A. Winter, ${ }^{4,5}$ and M. Lewenstein ${ }^{1,5}$ \\ ${ }^{1}$ ICFO_Institut de Ciències Fotòniques, The Barcelona Institute of Science and Technology, ES-08860 Castelldefels, Spain \\ ${ }^{2}$ Dahlem Center for Complex Quantum Systems, Freie Universität Berlin, D-14195 Berlin, Germany \\ ${ }^{3}$ Department of Physics and Astronomy, Southern Illinois University, Carbondale, Illinois 62901, USA \\ ${ }^{4}$ Física Teòrica: Informació i Fenòmens Quàntics, Universitat Autònoma de Barcelona, ES-08193 Bellaterra (Barcelona), Spain \\ ${ }^{5}$ ICREA_Institució Catalana de Recerca i Estudis Avançats, Passeig Lluis Companys 23, ES-O8010 Barcelona, Spain
}

(Received 30 March 2016; published 17 June 2016)

\begin{abstract}
Understanding the resource consumption in distributed scenarios is one of the main goals of quantum information theory. A prominent example for such a scenario is the task of quantum state merging, where two parties aim to merge their tripartite quantum state parts. In standard quantum state merging, entanglement is considered to be an expensive resource, while local quantum operations can be performed at no additional cost. However, recent developments show that some local operations could be more expensive than others: it is reasonable to distinguish between local incoherent operations and local operations which can create coherence. This idea leads us to the task of incoherent quantum state merging, where one of the parties has free access to local incoherent operations only. In this case the resources of the process are quantified by pairs of entanglement and coherence. Here, we develop tools for studying this process and apply them to several relevant scenarios. While quantum state merging can lead to a gain of entanglement, our results imply that no merging procedure can gain entanglement and coherence at the same time. We also provide a general lower bound on the entanglement-coherence sum and show that the bound is tight for all pure states. Our results also lead to an incoherent version of Schumacher compression: in this case the compression rate is equal to the von Neumann entropy of the diagonal elements of the corresponding quantum state.
\end{abstract}

DOI: 10.1103/PhysRevLett.116.240405

Introduction.-While coherence has long been known in classical physics as a fundamental wave property [1], in quantum mechanics coherent superposition is elevated to a universal principle governing all processes. Indeed, the fact that all matter exhibits wave behavior was first understood by de Broglie [2], which became the basis of the now standard formulation of quantum mechanics in Schrödinger's wave equation [3]. The universality of the superposition principle, i.e., the tenet that any two valid states of a system can be superposed to form a new valid state, marks a radical departure from classical physics. It is at the heart of the many counterintuitive features of quantum theory, perhaps most famously in Schrödinger's Gedankenexperiment with the cat [4]. Quantum entanglement can be considered a particular manifestation of coherence, and both of these nonclassical phenomena have led to extensive debates in the early days of quantum mechanics $[5,6]$.

While the study of the resource theory of entanglement has a long tradition $[7,8]$, the resource theory of quantum coherence was formulated only recently $[9,10]$, although other attempts in this direction were presented earlier [11-16]. The basis of any resource theory involves free states; these are states which can be created at no cost. In entanglement theory, these are all separable states. In coherence theory these are incoherent states [9], i.e., states which are diagonal in a fixed basis $|i\rangle$. The second important ingredient of any resource theory is that of free operations, i.e., operations which can be performed at no additional cost. In entanglement theory this is usually the set of local operations and classical communication, although other, more general sets such as separable operations $[17,18]$ and asymptotically nonentangling operations $[19,20]$ have also been considered. In coherence theory, free operations are called incoherent operations. These are precisely the quantum operations which have incoherent Kraus operators, i.e., $K_{i}|m\rangle \propto|n\rangle$, where $|m\rangle$ and $|n\rangle$ are elements of the incoherent basis [9].

Triggered by these recent developments, much effort is put into understanding the role of coherence as a resource in quantum theory [21-38]. Several new quantifiers of coherence have been proposed [39-52], and the dynamics of some of these quantities under noisy evolution has been investigated [53-57]. Several works also study maximally coherent states [58,59], the role of coherence in spin models $[60,61]$, the cohering power of quantum channels [62-64], and relations between coherence and other measures of quantumness [65-71]. Coherence also plays an important role in quantum thermodynamics [72-82], and its investigation in biological systems is an important step towards finding quantum phenomena in living objects [83-86]. Additionally, a distinction between "speakable" and "unspeakable" coherence was also introduced recently [87]. Here, we are describing coherence in a speakable sense, whereas unspeakable 
coherence is the resource captured in resource theories of asymmetry [15].

Contrary to entanglement, which inherently implies a scenario of at least two separated parties, the resource theory of coherence has initially been introduced for one party only. Very recently, there were several approaches to extend the notion of coherence to more than one party [53,65,68,70,88-93]. Here, we build on the methods presented in Refs. [89-91], aiming to study the interplay between entanglement and coherence in the task known as quantum state merging [94,95].

In standard quantum state merging, two parties-their names are traditionally Alice and Bob-share a mixed quantum state $\rho=\rho^{A B}$. Alice aims to send her part of the state to Bob via an additional quantum channel. The difficulty of the task arises from an extra requirement: the process has to be performed in such a way that the overall purification of the state remains intact. As was shown in Refs. [94,95], the singlet rate required for this process is equal to the conditional entropy $S(A \mid B)_{\rho}=$ $S\left(\rho^{A B}\right)-S\left(\rho^{B}\right)$, where $S(\rho)=-\operatorname{Tr}\left[\rho \log _{2} \rho\right]$ is the von Neumann entropy. To be precise, if the conditional entropy is positive, then merging is possible with singlets at rate $S(A \mid B)_{\rho}$, and merging is not possible if fewer singlets are available. Moreover, if the conditional entropy is negative, the process is possible without any entanglement. Apart from merging the state for free, Alice and Bob can additionally gain singlets at the rate $-S(A \mid B)_{\rho}$.

Here, we consider the task of incoherent quantum state merging. This task is very similar to standard quantum state merging, up to the fact that Bob has free access to incoherent operations only; i.e., he has to pay for operations which are not incoherent. There are at least two motivations for this: On the one hand, we would like to understand better the local quantum operations that Alice and, especially, Bob have to perform in merging. On the other hand, coherence seems to be the resource of choice to consider here, as entanglement and coherence are both resources of superposition, one in correlation, the other locally. Thus, while the cost of standard quantum state merging is quantified by the required entanglement rate $E$, the cost of incoherent quantum state merging will be quantified by a pair of entanglement and coherence rate $(E, C)$. Solving the problem of incoherent quantum state merging requires the characterization of all optimal pairs $(E, C)$. These are pairs of entanglement and coherence for which merging is possible, but neither entanglement nor coherence of the pair can be reduced.

At this point we note that the term "coherence" used in this Letter and other recent papers is, of course, also used in atomic and molecular physics, where coherences denote off-diagonal elements of the density matrix, typically on the basis of energy eigenstates. Note, however, that in quantum optics the term coherence is also used in the context of classical and quantum electrodynamics, where it describes the factorization property of certain correlation functions, ultimately related to the prominent Glauber-Sudarshan "coherent states" [96,97]. Off-diagonal elements of the density matrix, in the latter sense, are related rather to the "nonclassicality" of states of photons, phonons, bosons, etc. (cf. Refs. [98-100] and the references therein).

Incoherent quantum state merging.-We consider the scenario where three parties, Alice, Bob, and a referee, share a joint quantum state $\rho=\rho^{R A B}$. In the task of incoherent quantum state merging, Alice and Bob aim to merge their parts of the total state on Bob's side by using local quantumincoherent operations and classical communication (LQICC) [89]. Additionally, Alice and Bob have access to singlets at rate $E$ and maximally coherent states at rate $C$ on Bob's side.

In the following, we are interested in achievable pairs $(E, C)$. These are pairs combining coherence and entanglement for which the aforementioned task can be performed in the asymptotic scenario. Similar to standard quantum state merging [94,95], we consider the most general situation, where Alice and Bob can make catalytic use of entanglement and coherence [101]. We call $E_{i}$ the entanglement rate which is initially shared by Alice and Bob, and $E_{t}$ will be the final amount of entanglement between them. Similarly, $C_{i}$ and $C_{t}$ will be the initial and the final amount of Bob's local coherence. An entanglement-coherence pair $(E, C)$ is achievable if there exist numbers $E_{i}, E_{t}, C_{i}$, and $C_{t}$ with $E=E_{i}-E_{t}$ and $C=C_{i}-C_{t}$, such that, for any $\varepsilon>0$ and any $\delta>0$ for all sufficiently large integers $n \geq n_{0}$, there exists a LQICC protocol $\Lambda$ between Alice and Bob, such that

$$
\begin{aligned}
\| \Lambda\left[\rho_{i}^{\otimes n} \otimes \Phi_{2}^{\otimes\left\lfloor\left(E_{i}+\delta\right) n\right\rfloor} \otimes \Psi_{2}^{\otimes\left\lfloor\left(C_{i}+\delta\right) n\right\rfloor}\right] \\
-\rho_{t}^{\otimes n} \otimes \Phi_{2}^{\otimes\left[E_{t} n\right\rceil} \otimes \Psi_{2}^{\otimes\left[C_{t} n\right\rceil} \|_{1} \leq \epsilon .
\end{aligned}
$$

Here, $\rho_{i}=\rho^{R A B} \otimes|0\rangle\left\langle\left. 0\right|^{\tilde{B}}\right.$ is the total initial state, where $\tilde{B}$ is an additional particle in Bob's hands with the dimension $d_{\tilde{B}}=d_{A} . \quad\left|\Phi_{2}\right\rangle=\sqrt{\frac{1}{2}}(|00\rangle+|11\rangle) \quad$ is a maximally entangled two-qubit state shared by Alice and Bob, and $\left|\Psi_{2}\right\rangle=\sqrt{\frac{1}{2}}(|0\rangle+|1\rangle)$ is a maximally coherent single-qubit state on Bob's side. The target state $\rho_{t}=\rho^{R \tilde{B} B} \otimes|0\rangle\left\langle\left. 0\right|^{A}\right.$ is the same as $\rho_{i}$ up to relabeling the parties $A$ and $\tilde{B}$, and $\|M\|_{1}=\operatorname{Tr} \sqrt{M^{\dagger} M}$ is the trace norm.

The achievable region is a closed and convex set, owing to the timesharing principle [102,103]. Namely, on block length $n$ and for $0<p<1$, we can break the $n$ systems into two blocks of $k=\lfloor p n\rfloor$ and $\ell=\lceil(1-p) n\rceil$, and we can run a first protocol with asymptotic rate $\left(E_{1}, C_{1}\right)$ on the $k$ block, and a second protocol with asymptotic rate $\left(E_{2}\right.$, $C_{2}$ ) on the $\ell$ block. The tensor product of these protocols is evidently an asymptotically error-free merging protocol, and it achieves the rate pair $(E, C)=\left[p E_{1}+(1-p) E_{2}\right.$, $\left.p C_{1}+(1-p) C_{2}\right]$.

As in standard quantum state merging, the quantities $E$ and $C$ can be positive or negative. If $E(C)$ is positive, it 
means that the merging procedure consumes entanglement (coherence) at the rate $E(C)$. If the corresponding quantity is negative, the process can be performed without the corresponding resource, and singlets (maximally coherent states) are gained as well. Crucially, as we will see later in this Letter, the latter gain is not possible for both entanglement and coherence at the same time: if entanglement is gained in the process, coherence has to be consumed, and vice versa.

Clearly, if a pair $(E, C)$ is achievable, then any other pair $\left(E^{\prime}, C^{\prime}\right)$ is also achievable for $E^{\prime} \geq E$ and $C^{\prime} \geq C$. A pair $(E, C)$ will be called optimal if it is achievable and if the pairs $\left(E, C^{\prime}\right)$ and $\left(E^{\prime}, C\right)$ are not achievable for any $C^{\prime}<C$ and $E^{\prime}<E$. Since via LQICC operations a singlet can be converted into a maximally coherent state on Bob's side [89], with every achievable pair $(E, C),(E+t, C-t)$ is also achievable for $t>0$. Thus, it is always possible to perform incoherent merging with $C=0$, and the corresponding optimal pair will be denoted $\left(E_{0}, 0\right)$. Another important pair is the one with the minimal amount of entanglement, $E_{\min }$, among all protocols. We denote it $\left(E_{\min }, C_{\max }\right)$ since it also has the maximal amount of coherence among all optimal pairs [104].

A full solution of incoherent quantum state merging implies determining all optimal pairs for a given tripartite state. The following proposition provides a bound on the entanglement-coherence sum $E+C$.

Proposition 1.-Given a tripartite quantum state $\rho=\rho^{R A B}$, any achievable pair $(E, C)$ fulfills the following inequality:

$$
E+C \geq S\left(\mathrm{id}^{R} \otimes \Delta^{A B}[\rho]\right)-S\left(\mathrm{id}^{R A} \otimes \Delta^{B}[\rho]\right),
$$

where $\Delta^{X}[\rho]$ denotes full decoherence of the state $\rho$ in the incoherent basis of a (possibly multipartite) subsystem $X$ : $\Delta^{X}[\rho]=\sum_{i}|i\rangle\left\langle\left. i\right|^{X} \rho \mid i\right\rangle\left\langle\left. i\right|^{X}\right.$. We refer the reader to Sec. I of the Supplemental Material [105] for the proof, which is based on monotonicity of QI relative entropy under LQICC operations [89].

It is instructive to compare these results to standard quantum state merging, as presented in Refs. [94,95]. In standard quantum state merging, the entanglement rate required for merging a pure state $\psi^{R A B}$ is given by the conditional entropy of the reduced state $\rho^{A B}$, which can be either positive or negative. In the negative case, quantum state merging is possible without entanglement and additional singlets are produced. Since the right-hand side of Eq. (2) cannot be negative, it follows that the sum $E+C$ is also non-negative. While each of the quantities $E$ or $C$ can still be negative individually, they cannot both be negative at the same time. Thus, there is no merging procedure where entanglement and coherence are gained simultaneously. This statement is true for all mixed states $\rho^{R A B}$.

Having presented the general framework, we will now focus on the situation where the total state is pure. Note that understanding of the pure-state scenario also gives insights for general mixed states. Specifically, if a pair $(E, C)$ is achievable for a pure state $|\psi\rangle^{R A B}$, the same pair is also achievable for any state $\rho^{R A B}$ with the same reduction, such that $\rho^{A B}=\operatorname{Tr}_{R}\left[\psi^{R A B}\right]$.

Incoherent merging of pure states.-We will now consider incoherent quantum state merging for general pure states. By state merging [95,110], we have $E \geq E_{\min }=$ $S(A \mid B)_{\rho}$ with the reduced state $\rho=\rho^{A B}$. Moreover, for pure states Proposition 1 reduces to $E+C \geq S(A \mid B)_{\bar{\rho}}$, with the dephased state $\bar{\rho}=\Delta^{A B}\left[\rho^{A B}\right]$. As we will see in the following theorem, this bound is saturated.

Theorem 2.-Any pure state $|\psi\rangle^{R A B}$ can be merged with the optimal pair $\left(E_{0}, C=0\right)$, where $E_{0}=S\left(\bar{\rho}^{A B}\right)-S\left(\bar{\rho}^{B}\right)$.

We refer to Sec. II of the Supplemental Material [105] for the proof, which is based on an adaptation of the SlepianWolf distributed compression of the decohered-classicalsource. Note that $\bar{\rho}^{A B}$ is a classical state, and its conditional entropy, according to the Slepian-Wolf theorem [111], is precisely the amount of classical communication required to inform Bob about Alice's register. In fact, the proof of this theorem in Sec. II of the Supplemental Material [105] uses the Slepian-Wolf protocol as a building block.

The above theorem implies that, for pure states $\psi^{R A B}$, the minimal entanglement-coherence sum $E+C$ required for merging is equal to the conditional entropy of the decohered state $\bar{\rho}^{A B}$. We also mention that, for pure states of the form $|\psi\rangle^{R A} \otimes|0\rangle^{B}$, the procedure described here can be seen as the incoherent version of Schumacher compression [112]. Specifically, Theorem 2 proves that any state $\rho$ can be faithfully compressed at the rate $S(\Delta[\rho])$, under the assumption that the decompression is performed with incoherent operations only.

A final comment is in order concerning the applicability of Proposition 1 and Theorem 2 to different operational classes. Beyond the incoherent operations considered in this Letter, one can consider the more general class of "maximal" incoherent operations (MIO), which consists of all non-coherence-generating maps $[10,113]$. As we discuss in Sec. I of the Supplemental Material [105], the lower bound of Proposition 1 holds as well for MIO. On the achievability end, the rate of Theorem 2 is still achievable when Bob is limited to so-called strictly incoherent operations [10,32], and even if he is further restricted to the class of physical incoherent operations [49]. Also, Alice's measurement in Theorem 2 can always be made incoherent since the protocol is one way, with her final state being incoherent. Thus, our result also applies to the scenario of bipartite local incoherent operations and classical communications [90,91].

Coherence-entanglement trade-off.-The development so far revealed some facts about the landscape of the achievable pairs $(E, C)$ for incoherent merging of a state $\rho^{R A B}$. Most importantly, there are two inaccessible regions 
given by the inequalities $E+C \geq S\left(\Delta^{A B}[\rho]\right)-S\left(\Delta^{B}[\rho]\right)$ and $E \geq E_{\min }$. For a pure state, these simplify to $E+C \geq$ $S(A \mid B)_{\bar{\rho}}$ and $E \geq S(A \mid B)_{\rho}$, and the lower bound is tight, as $\left[E=E_{0}=S(A \mid B)_{\bar{\rho}}, C=0\right]$ is achievable. Furthermore, since with every achievable pair $(E, C)$ it is true that $(E+t, C-t)$ is also achievable for $t>0$, we find a boundary of the achievable region in the line of slope -1 from $\left(E_{0}, 0\right)$ to the right; see Fig. 1. We do not know at this point whether this boundary line continues with slope -1 also to the left of that point. The biggest open question is the characterization of $C_{\max }$, which is the coherence rate required for the minimum possible entanglement rate $E_{\min }$. Naturally, if we could show that $\left(E=E_{\min }, C=E_{0}-E_{\min }\right)$ is achievable, we would have characterized the entire achievable region, showing that it is delimited by the two above mentioned linear inequalities. On the other hand, it is quite conceivable that, in general, $C_{\max } \gg E_{0}-E_{\min }$.

We are now going to present an example indicative of the second option inspired by the "flower states" [114]:

$$
|\psi\rangle^{R A B}=\frac{1}{\sqrt{2 d}} \sum_{i=0}^{1} \sum_{j=1}^{d}\left(U_{i}^{\top}|j\rangle\right)^{R}|i\rangle^{A}|j\rangle^{B},
$$

where, for definitiveness, $U_{0}=\mathbb{1}, U_{1}=\mathrm{QFT}$ is the quantum Fourier transform. One checks to see that, for this family of states, $E_{0}=1$ (attained by simply teleporting Alice's qubit) and $E_{\min }=0$. Indeed, there is a simple exact

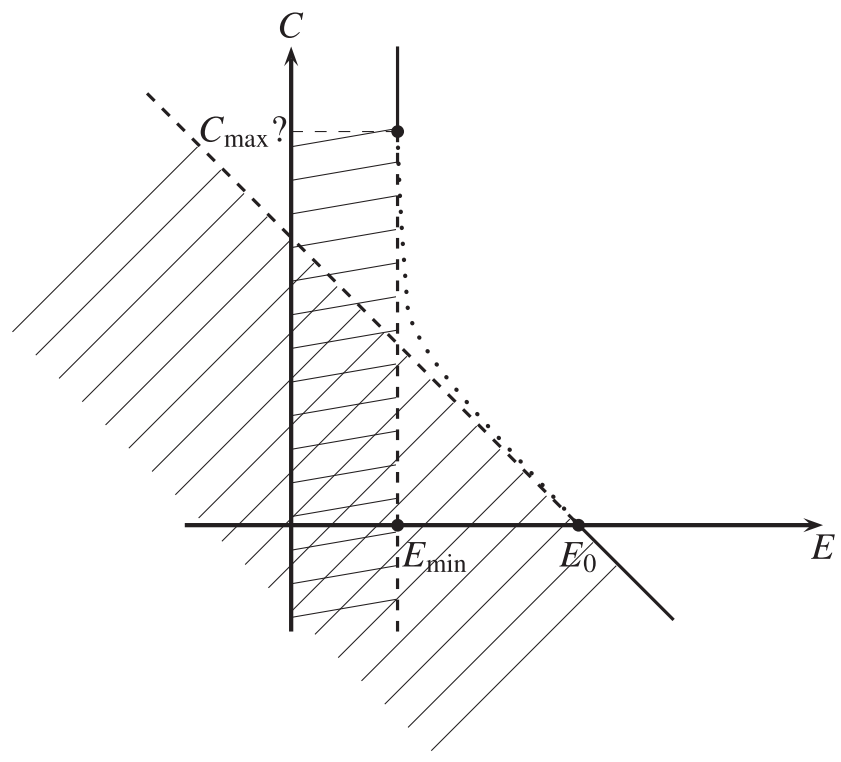

FIG. 1. The achievable region and known bounds for coherence and entanglement required to merge a general pure state $\psi^{R A B}$. The shaded regions to the left and below the straight lines are ruled out. The solid line of slope -1 to the right, downward from $\left(E_{0}, 0\right)$, as well as the solid vertical line upward from $\left(E_{\min }, C_{\max }\right)$, are part of the boundary of the achievable region. The dotted curve connecting these two points represents $C(E)$, the general form of which is not known, however. The quantities $E_{0}$ and $E_{\min }$ are given as $E_{0}=S(A \mid B)_{\bar{\rho}}, E_{\min }=S(A \mid B)_{\rho}$. merging protocol not using any entanglement, which consists of Alice measuring in the computational basis and communicating $i$ to Bob. Bob, in turn, applies $U_{i}^{\dagger}$, after which he is left with the maximally entangled state $\left|\Phi_{d}\right\rangle^{R B}$ with the reference; now he creates the state $|+\rangle^{\tilde{B}}=$ $(1 / \sqrt{2})(|0\rangle+|1\rangle)$ and recovers the state $|\psi\rangle^{R \tilde{B} B}$ by the controlled unitary $|0\rangle\left\langle 0\left|\otimes U_{0}+\right| 1\right\rangle\langle 1| \otimes U_{1}$. Note that while $U_{0}$ is trivial, $U_{1}$ requires a large amount of coherence to be implemented. Indeed, the previous procedure of Bob requires asymptotically a rate of $1+\frac{1}{2} \log d$ of coherence. Conversely, we have the following lower bound.

Theorem 3.-Merging the state in Eq. (3) via one-way LQICC without any initial entanglement-i.e., not only $E_{i}=0$ but also $\delta=0$ in Eq. (1)-requires a rate of coherence of at least $C \geq 1+\frac{1}{2} \log d \gg 1$.

We refer to Sec. III of the Supplemental Material [105] for the proof. While we proved the theorem for the case where classical communication only goes in one direction, it is reasonable to believe that this result can be extended to arbitrary LQICC protocols. We also note another limitation of the result: our proof covers only the case that entanglement is exactly zero initially. It is not clear if this result also applies when considering a more general merging procedure where entanglement vanishes only in the asymptotic limit. Nevertheless, this result provides strong evidence that, in the task of quantum state merging, it is possible to save a large amount of local coherence by using a little extra entanglement.

In Sec. IV of the Supplemental Material [105], we also study a family of mixed fully separable states of the form $\rho=\sum_{i, j} p_{i j}|i j\rangle\left\langle\left. i j\right|^{R} \otimes \mid \psi_{i j}\right\rangle\left\langle\left.\psi_{i j}\right|^{A} \otimes \mid i\right\rangle\left\langle\left. i\right|^{B}\right.$, where the states $\left|\psi_{i j}\right\rangle$ are mutually orthogonal for different $j$ 's, i.e., $\left\langle\psi_{i j} \mid \psi_{i k}\right\rangle=\delta_{j k}$. As shown in the Supplemental Material [105], for all of these states, all optimal pairs are given by $(E, C)=\left(a C_{\max },[1-a] C_{\max }\right)$, with $a \geq 0$ and $C_{\max }=\sum_{i, j} p_{i j} S\left(\Delta\left(\psi_{i j}\right)\right)$.

Conclusions. - In this Letter we introduced and studied the task of incoherent quantum state merging. This task is the same as standard quantum state merging, up to the fact that one of the parties has free access to local incoherent operations only and has to consume a coherent resource for more general operations. The amount of resources needed for merging is quantified by an entanglement-coherence pair $(E, C)$. In general, we showed that the entanglementcoherence sum $E+C$ is non-negative, which means that no merging procedure can gain entanglement and coherence at the same time. For pure states, we gave a protocol of incoherent quantum state merging by finding the minimal entanglement-coherence sum $E+C$, which turns out to be the conditional entropy of the decohered state $\bar{\rho}^{A B}$.

Our results include an incoherent version of Schumacher compression. Specifically, if we require that the decompression is performed via incoherent operations only, then the optimal compression rate is given by $S(\Delta(\rho))$. This rate 
is, in general, larger than the standard compression rate $S(\rho)$, which results from the fact that coherence is required for the decompression in the standard case.

We have also made first steps towards an understanding of the precise trade-off between entanglement and coherence for the task of LQICC merging. While this remains a major open problem in general, we have given strong indications that in certain situations the equivalent of one ebit can be an arbitrary amount of coherence, which we could prove in a setting of one-way LQICC and a situation where we want to reduce the available entanglement exactly (and not only asymptotically) to zero.

Another open question is the relation of LQICC merging to the results presented in Ref. [117]. The authors of Ref. [117] specifically study the work cost for erasing a system $A$ which is (quantum) correlated with another observer $B$ in an environment at temperature $T$. As was shown in Ref. [117], this work cost is bounded above by $S(A \mid B) k T \ln (2)$, where $k$ is the Boltzmann constant. At this point, it is natural to ask whether our results can be applied to understanding the role of coherence in the erasure process. We leave these questions for future research.

We thank Jens Eisert and Christian Majenz for discussion, and Martin B. Plenio for his comments on the manuscript. We acknowledge financial support from the Alexander von Humboldt Foundation, the John Templeton Foundation, the European Commission (QUIC and STREP RAQUEL), the European Research Council (AdG OSYRIS and AdG IRQUAT), the U.S. National Science Foundation (CAREER Grant No. 1352326), the Spanish MINECO (Grants No. FIS2013-46768, No. FIS2008-01236, No. FIS2013-40627-P, and Severo Ochoa Excellence Grant No. SEV-2015-0522) with the support of FEDER funds, the Generalitat de Catalunya (Grants No. 2014SGR-874 and No. 2014-SGR-966), and Fundació Privada Cellex.

*streltsov.physics@gmail.com

[1] T. Young, A Course of Lectures on Natural Philosophy and the Mechanical Arts, Vol. 2 (J. Johnson, London, 1807).

[2] L. de Broglie, J. Phys. Radium 2, 33 (1922); Nature (London) 112, 540 (1923); Ann. de Physique 3, 22 (1925).

[3] E. Schrödinger, Ann. Phys. (Berlin) 384, 361 (1926); 384, 489 (1926); 384, 734 (1926); 385, 437 (1926); 386, 109 (1926); Naturwissenschaften 14, 664 (1926); Phys. Rev. 28, 1049 (1926).

[4] E. Schrödinger, Naturwissenschaften 23, 807 (1935); 23, 823 (1935); 23, 844 (1935).

[5] A. Einstein, B. Podolsky, and N. Rosen, Phys. Rev. 47, 777 (1935).

[6] N. Bohr, Phys. Rev. 48, 696 (1935).

[7] M. B. Plenio and S. Virmani, Quantum Inf. Comput. 7, 1 (2007).

[8] R. Horodecki, P. Horodecki, M. Horodecki, and K. Horodecki, Rev. Mod. Phys. 81, 865 (2009).
[9] T. Baumgratz, M. Cramer, and M. B. Plenio, Phys. Rev. Lett. 113, 140401 (2014).

[10] A. Winter and D. Yang, Phys. Rev. Lett. 116, 120404 (2016).

[11] J. Aberg, arXiv:quant-ph/0612146.

[12] G. Gour and R. W. Spekkens, New J. Phys. 10, 033023 (2008).

[13] G. Gour, I. Marvian, and R. W. Spekkens, Phys. Rev. A 80, 012307 (2009).

[14] F. Levi and F. Mintert, New J. Phys. 16, 033007 (2014).

[15] I. Marvian and R. W. Spekkens, New J. Phys. 15, 033001 (2013).

[16] I. Marvian and R. W. Spekkens, Phys. Rev. A 90, 062110 (2014).

[17] E. M. Rains, arXiv:quant-ph/9707002.

[18] V. Vedral and M. B. Plenio, Phys. Rev. A 57, 1619 (1998).

[19] F. Brandão and M. B. Plenio, Nat. Phys. 4, 873 (2008).

[20] F. Brandão and M. B. Plenio, Commun. Math. Phys. 295, 829 (2010).

[21] J.-J. Chen, J. Cui, and H. Fan, arXiv:1509.03576.

[22] S. Cheng and M. J. W. Hall, Phys. Rev. A 92, 042101 (2015).

[23] D. Mondal, C. Datta, and S. Sazim, arXiv:1506.03199.

[24] P. Deb, Quantum Inf. Process. 15, 1629 (2016).

[25] S. Du, Z. Bai, and Y. Guo, Phys. Rev. A 91, 052120 (2015).

[26] M. N. Bera, T. Qureshi, M. A. Siddiqui, and A. K. Pati, Phys. Rev. A 92, 012118 (2015).

[27] X. Liu, Z. Tian, J. Wang, and J. Jing, Ann. Phys. (Amsterdam) 366, 102 (2016).

[28] H. Li, J. Zou, W.-L. Yu, B.-M. Xu, J.-G. Li, and B. Shao, Phys. Rev. E 89, 052132 (2014).

[29] K. von Prillwitz, Ł. Rudnicki, and F. Mintert, Phys. Rev. A 92, 052114 (2015).

[30] S. Du, Z. Bai, and X. Qi, arXiv:1504.02862.

[31] M. Hillery, Phys. Rev. A 93, 012111 (2016).

[32] B. Yadin, J. Ma, D. Girolami, M. Gu, and V. Vedral, arXiv:1512.02085.

[33] K. Bu, U. Singh, and J. Wu, Phys. Rev. A 93, 042326 (2016).

[34] J. M. Matera, D. Egloff, N. Killoran, and M. B. Plenio, arXiv:1512.07486.

[35] U. Singh, L. Zhang, and A. K. Pati, Phys. Rev. A 93, 032125 (2016).

[36] Y.-R. Zhang, L.-H. Shao, Y. Li, and H. Fan, Phys. Rev. A 93, 012334 (2016).

[37] U. Singh, M. N. Bera, H. S. Dhar, and A. K. Pati, Phys. Rev. A 91, 052115 (2015).

[38] J. Chen, N. Johnston, C.-K. Li, and S. Plosker, arXiv:1601.06269.

[39] D. Girolami, Phys. Rev. Lett. 113, 170401 (2014).

[40] S. Du and Z. Bai, arXiv:1505.00151.

[41] X. Yuan, H. Zhou, Z. Cao, and X. Ma, Phys. Rev. A 92, 022124 (2015).

[42] D. P. Pires, L. C. Céleri, and D. O. Soares-Pinto, Phys. Rev. A 91, 042330 (2015).

[43] X. Qi, Z. Bai, and S. Du, arXiv:1505.07387.

[44] J. Xu, Phys. Rev. A 93, 032111 (2016).

[45] B. Yadin and V. Vedral, Phys. Rev. A 93, 022122 (2016).

[46] S. Rana, P. Parashar, and M. Lewenstein, Phys. Rev. A 93, 012110 (2016). 
[47] M. Allegra, P. Giorda, and S. Lloyd, Phys. Rev. A 93, 042312 (2016).

[48] R. Chandrashekar, P. Manikandan, J. Segar, and T. Byrnes, Phys. Rev. Lett. 116, 150504 (2016).

[49] E. Chitambar and G. Gour, arXiv:1602.06969.

[50] A. Streltsov, arXiv:1511.08346.

[51] C. Napoli, T. R. Bromley, M. Cianciaruso, M. Piani, N. Johnston, and G. Adesso, Phys. Rev. Lett. 116, 150502 (2016).

[52] M. Piani, M. Cianciaruso, T. R. Bromley, C. Napoli, N. Johnston, and G. Adesso, Phys. Rev. A 93, 042107 (2016).

[53] T. R. Bromley, M. Cianciaruso, and G. Adesso, Phys. Rev. Lett. 114, 210401 (2015).

[54] U. Singh, M. N. Bera, A. Misra, and A. K. Pati, arXiv:1506.08186.

[55] T. Chanda and S. Bhattacharya, arXiv:1505.05810.

[56] Y.-J. Zhang, W. Han, Y.-J. Xia, Y.-M. Yu, and H. Fan, Sci. Rep. 5, 13359 (2015).

[57] I. A. Silva, A. M. Souza, T. R. Bromley, M. Cianciaruso, R. S. Sarthour, I. S. Oliveira, R. Lo Franco, E. R. deAzevedo, D. O. Soares-Pinto, and G. Adesso, arXiv:1511.01971.

[58] Y. Peng, Y. Jiang, and H. Fan, Phys. Rev. A 93, 032326 (2016).

[59] Z. Bai and S. Du, arXiv:1503.07103.

[60] G. Karpat, B. Çakmak, and F. F. Fanchini, Phys. Rev. B 90, 104431 (2014).

[61] B. Çakmak, G. Karpat, and F. F. Fanchini, Entropy 17, 790 (2015).

[62] A. Mani and V. Karimipour, Phys. Rev. A 92, 032331 (2015).

[63] K. Bu, L. Zhang, and J. Wu, arXiv:1509.09109.

[64] M. García-Díaz, D. Egloff, and M. B. Plenio, arXiv:1510.06683.

[65] A. Streltsov, U. Singh, H. S. Dhar, M. N. Bera, and G. Adesso, Phys. Rev. Lett. 115, 020403 (2015).

[66] N. Killoran, F. E. S. Steinhoff, and M. B. Plenio, Phys. Rev. Lett. 116, 080402 (2016).

[67] J. Ma, B. Yadin, D. Girolami, V. Vedral, and M. Gu, Phys. Rev. Lett. 116, 160407 (2016).

[68] Y. Yao, X. Xiao, L. Ge, and C. P. Sun, Phys. Rev. A 92, 022112 (2015).

[69] Z. Xi, Y. Li, and H. Fan, Sci. Rep. 5, 10922 (2015).

[70] X. Hu and H. Fan, arXiv:1508.01978.

[71] D. Girolami and B. Yadin, arXiv:1509.04131.

[72] P. Skrzypczyk, A. J. Short, and S. Popescu, Nat. Commun. 5, 4185 (2014).

[73] J. Åberg, Phys. Rev. Lett. 113, 150402 (2014).

[74] M. Lostaglio, D. Jennings, and T. Rudolph, Nat. Commun. 6, 6383 (2015).

[75] M. Lostaglio, K. Korzekwa, D. Jennings, and T. Rudolph, Phys. Rev. X 5, 021001 (2015).

[76] K. Korzekwa, M. Lostaglio, J. Oppenheim, and D. Jennings, New J. Phys. 18, 023045 (2016).

[77] P. Kammerlander and J. Anders, Sci. Rep. 6, 22174 (2016).

[78] G. Vacanti, C. Elouard, and A. Auffeves, arXiv:1503.01974.

[79] G. Gour, M. P. Müller, V. Narasimhachar, R. W. Spekkens, and N. Y. Halpern, Phys. Rep. 583, 1 (2015).

[80] A. L. Malvezzi, G. Karpat, B. Çakmak, F. F. Fanchini, T. Debarba, and R. O. Vianna, arXiv:1602.03731 [Phys. Rev. B (to be published)].
[81] S. Bhattacharya, S. Banerjee, and A. K. Pati, arXiv: 1601.04742.

[82] Y. Yang and G. Chiribella, arXiv:1502.00259.

[83] S. Lloyd, J. Phys. Conf. Ser. 302, 012037 (2011).

[84] C.-M. Li, N. Lambert, Y.-N. Chen, G.-Y. Chen, and F. Nori, Sci. Rep. 2, 885 (2012).

[85] S. F. Huelga and M. B. Plenio, Contemp. Phys. 54, 181 (2013).

[86] V. Singh Poonia, D. Saha, and S. Ganguly, arXiv:1408.1327.

[87] I. Marvian and R. W. Spekkens, arXiv:1602.08049.

[88] X. Hu, A. Milne, B. Zhang, and H. Fan, Sci. Rep. 6, 19365 (2016).

[89] E. Chitambar, A. Streltsov, S. Rana, M. N. Bera, G. Adesso, and M. Lewenstein, Phys. Rev. Lett. 116, 070402 (2016).

[90] E. Chitambar and M.-H. Hsieh, arXiv:1509.07458.

[91] A. Streltsov, S. Rana, M. Nath Bera, and M. Lewenstein, arXiv:1509.07456.

[92] A. Kumar, arXiv:1508.00262.

[93] D. Mondal, T. Pramanik, and A. K. Pati, arXiv:1508.03770.

[94] M. Horodecki, J. Oppenheim, and A. Winter, Nature (London) 436, 673 (2005).

[95] M. Horodecki, J. Oppenheim, and A. Winter, Commun. Math. Phys. 269, 107 (2007).

[96] R. J. Glauber, Phys. Rev. 131, 2766 (1963).

[97] E. C. G. Sudarshan, Phys. Rev. Lett. 10, 277 (1963).

[98] J. K. Korbicz, J. I. Cirac, J. Wehr, and M. Lewenstein, Phys. Rev. Lett. 94, 153601 (2005).

[99] W. Vogel and D.-G. Welsch, Quantum Optics, 3rd ed. (Wiley-VCH, Weinheim, 2006).

[100] W. P. Schleich, Quantum Optics in Phase Space, 1st ed. (Wiley-VCH, Weinheim, 2001).

[101] In general, a process makes catalytic use of a resource if it starts with a certain amount of the resource $R_{1}$ and ends with some nonzero amount, $R_{2}>0$.

[102] T. M. Cover and J. A. Thomas, Elements of Information Theory, 2nd Ed., Wiley Series in Telecommunications and Signal Processing (Wiley InterScience, New York, 2006).

[103] I. Csiszár and J. Körner, Information Theory: Coding Theorems for Discrete Memoryless Systems, 2nd ed. (Cambridge University Press, Cambridge, England, 2011).

[104] To see that the pair $\left(E_{\min }, C_{\max }\right)$ has the maximal amount of coherence among all optimal pairs, consider two optimal pairs, $(E, C)$ and $\left(E^{\prime}, C^{\prime}\right)$, with $C<C^{\prime}$. Then, by optimality, it must be that $E>E^{\prime}$. Suppose now that there existed a pair $(\tilde{E}, \tilde{C})$ with $\tilde{C}>C_{\max }$. By the aforementioned argument, it follows that $\tilde{E}<E_{\min }$, which is a contradiction.

[105] See Supplemental Material at http://link.aps.org/ supplemental/10.1103/PhysRevLett.116.240405, which includes Refs. [106-109], for quantum teleportation, Maassen-Uffink uncertainty relation, and results on entanglement-breaking channels.

[106] C. H. Bennett, G. Brassard, C. Crépeau, R. Jozsa, A. Peres, and W. K. Wootters, Phys. Rev. Lett. 70, 1895 (1993).

[107] H. Maassen and J. B. M. Uffink, Phys. Rev. Lett. 60, 1103 (1988).

[108] P. W. Shor, J. Math. Phys. (N.Y.) 43, 4334 (2002).

[109] C. King, Quantum Inf. Comput. 3, 186 (2003). 
[110] M. Horodecki, P. Horodecki, R. Horodecki, J. Oppenheim, A. Sen(De), U. Sen, and B. Synak-Radtke, Phys. Rev. A 71, 062307 (2005).

[111] D. Slepian and J. Wolf, IEEE Trans. Inf. Theory 19, 471 (1973).

[112] B. Schumacher, Phys. Rev. A 51, 2738 (1995).

[113] F. G. S. L. Brandão and G. Gour, Phys. Rev. Lett. 115, 070503 (2015).

[114] Note that the flower states presented in Ref. [115] are obtained by purifying the information locking states of
Ref. [116], and their results are mathematically equivalent due to the duality between the entanglement of formation and the Henderson-Vedral information.

[115] K. Horodecki, M. Horodecki, P. Horodecki, and J. Oppenheim, Phys. Rev. Lett. 94, 200501 (2005).

[116] D. P. DiVincenzo, M. Horodecki, D. W. Leung, J. A. Smolin, and B. M. Terhal, Phys. Rev. Lett. 92, 067902 (2004).

[117] L. del Rio, J. Åberg, R. Renner, O. Dahlsten, and V. Vedral, Nature (London) 474, 61 (2011). 